\title{
COMMENTARY
}

\section{Copeptin in aneurysmal subarachnoid hemorrhage}

\author{
Rafael J Tamargo* \\ See related research by Zhu et al., http://ccforum.com/content/15/6/R288
}

\begin{abstract}
Copeptin is a peptide derived from preprovasospression along with arginine vasospressin. In the setting of aneurysmal subarachnoid hemorrhage (SAH), elevated serum copeptin levels correlate with vasospasm, inpatient mortality, mortality at 1 year, and poor functional outcome at 1 year. The potential role of serum copeptin levels in the management of patients with aneurysmal SAH is promising and should be explored further.
\end{abstract}

The study by Zhu and colleagues [1], of Zhejiang University in China, in the previous issue of Critical Care is of particular interest because they report that in a population of 303 patients with aneurysmal subarachnoid hemorrhage (SAH), elevated serum copeptin levels correlated not only with poor outcomes and higher mortality but, more importantly, with vasospasm during the subacute period. A growing understanding of the pathophysiology of aneurysmal SAH has prompted efforts to identify serum markers that can predict outcomes in these patients. Since the most important treatable determinant of poor outcome after aneurysmal SAH is the delayed neurological deterioration observed 4 to 14 days after SAH (previously identified as 'vasospasm' [2] but now preferentially labeled 'delayed cerebral injury' or 'delayed cerebral ischemia'), several investigators have attempted to identify serum markers that can predict vasospasm in particular and poor functional outcome in general. Serum markers predictive of vasospasm, however, have proved elusive. For instance, the recognition of the important role of an inflammatory injury after aneurysmal SAH [3] led to an exploration of the predictive value

\footnotetext{
*Correspondence: rtamarg@jhmi.edu

Department of Neurosurgery, Johns Hopkins University School of Medicine, The Johns Hopkins Hospital, 600 N. Wolfe Street, Meyer 8-181, Baltimore, MD 21287 , USA
}

of inflammatory markers. Recently, Juvela and colleagues [4], of the University of Helsinki in Finland, reported that elevated serum levels of C-reactive protein (an acutephase inflammatory marker) are predictive of poor outcome at 3 months after aneurysmal SAH but not of delayed cerebral ischemia or infarction ('vasospasm'). Similarly, the recognition of the adverse changes initiated by the contact of blood with the extravascular matrix, which cause impaired coagulation and fibrinolysis, led to a prior exploration by the same group of the predictive value of elevated serum D-dimer levels. In 2006, Juvela and Siironen [5] reported that elevated serum D-dimer levels are predictive of poor outcome at 3 months after aneurysmal SAH but, again, not of delayed cerebral ischemia or infarction ('vasospasm'). In the study by Zhu and colleagues, elevated serum copeptin levels were predictive of vasospasm as well as poor outcome.

Under stress, the hypothalamic-pituitary axis produces stress hormones such as corticotropin-releasing homone and the nonapeptide arginine vasospressin (AVP). Among the many effects of AVP are water retention in the kidneys and increased blood pressure, the latter of which is mediated mostly by vasoconstriction. Efforts to measure serum levels of AVP in a clinical setting, however, have been limited by the fact that it is unstable at room temperature and is cleared rapidly from plasma [6]. The 164-amino acid precursor peptide of AVP, preprovasopresssin, is broken down into three peptides: AVP, neurophysin II, and copeptin [7]. Since the larger copeptin is produced in an equimolar ratio with AVP and is easier to measure in serum, it can be a surrogate marker for AVP. Elevated copeptin levels have been correlated with poor outcomes in ischemic stroke, intraparenchymal hemorrhage, and brain trauma as well as sepsis, pneumonia, and myocardial infarction [6].

In their retrospective study [1], Zhu and colleagues extend this work on copeptin to aneurysmal SAH and demonstrate convincingly that elevated serum copeptin levels correlate with vasospasm, inpatient mortality, mortality at 1 year, and poor functional outcome at 1 year. Serum copeptin levels were measured on admission in 303 patients. As controls, 150 healthy, gender- and 
age-matched volunteers were used. Vasospasm was confirmed by computed tomography angiography and catheter angiography in all cases. The epidemiological profile and outcomes of the 303 study patients - $10.6 \%$ inpatient mortality, 13.9\% 1-year mortality, 29.7\% 1-year poor outcomes, and $43.2 \%$ incidence of vasospasm - are similar to those of patients in larger series. In this study, World Federation of Neurological Surgeons (WFNS) grade, modified Fisher grade, and copeptin levels (but not aneurysm size, vasospasm, clot thickness, or ischemia by computed tomography) were associated with increased mortality and poor outcomes by multivariate analysis. The predictive value of copeptin was similar to that of the WFNS score in terms of 1-year mortality but was lower than that of the WFNS score in terms of vasospasm. It is somewhat disappointing that, in a combined logistic-regression analysis, copeptin did not improve upon the area under the curve of the WFNS score in a statistically significant fashion. Nevertheless, the potential role of serum copeptin levels in the management of patients with aneurysmal SAH is promising and should be explored further.

\section{Abbreviations}

AVP, arginine vasospressin; SAH, subarachnoid hemorrhage; WFNS, World Federation of Neurological Surgeons.
Competing interests

The author declares that he has no competing interests.

Published: 10 January 2012

References

1. Zhu XD, Chen JS, Zhou F, Liu QC, Chen G, Zhang JM: Detection of copeptin in peripheral blood of patients with aneurysmal subarachnoid hemorrhage. Crit Care 2011, 15:R288.

2. Tamargo RJ, Walter KA, Oshiro EM: Aneurysmal subarachnoid hemorrhage: prognostic features and outcomes. New Horiz 1997, 5:364-375.

3. Chaichana KL, Pradilla G, Huang J, Tamargo RJ: Role of inflammation (leukocyte-endothelial cell interactions) in vasospasm after subarachnoid hemorrhage. World Neurosurg 2010, 73:22-41.

4. Juvela S, Kuhmonen J, Siironen J: C-reactive protein as predictor for poor outcome after aneurysmal subarachnoid haemorrhage. Acta Neurochir (Wien) 2011 Dec 3. [Epub ahead of print].

5. Juvela S, Siironen J: D-dimer as an independent predictor for poor outcome after aneurysmal subarachnoid hemorrhage. Stroke 2006, 37:1451-1456.

6. Katan M, Christ-Crain M: The stress hormone copeptin: a new prognostic biomarker in acute illness. Swiss Med Wkly 2010, 140:w13101.

7. Morgenthaler NG, Struck J, Alonso C, Bergmann A: Assay for the measurement of copeptin, a stable peptide derived from the precursor of vasopressin. Clin Chem 2006, 52:112-119.

doi:10.1186/cc10594

Cite this article as: Tamargo RJ: Copeptin in aneurysmal subarachnoid hemorrhage. Critical Care 2012, 16:103. 